\title{
Nonlinear slow light propagation in photonic crystal slab waveguides: theory and practical issues
}

\author{
Panagiotis. Kanakis ${ }^{\mathrm{a}}$, Thomas Kamalakis ${ }^{\mathrm{b}}$, Thomas Sphicopoulos ${ }^{\mathrm{a}}$ \\ ${ }^{a}$ Dept. of Informatics and Telecom., National and Kapodistrian Univ./Athens, Panepistimioupolis, \\ Athens, Greece GR157 84; \\ ${ }^{b}$ Dept. of Informatics and Telematics, Harokopio Univ./Athens, 89 Harokopou str., Athens, \\ Greece GR176 71
}

\begin{abstract}
In this paper, we consider the propagation of slow light optical pulses inside photonic crystal slab waveguides (PCSW) both from a theoretical and an application point-of-view. The numerical model used relies on a nonlinear envelope propagation equation that includes the effects of second and third order dispersion, optical losses and self phase modulation. Pulse propagation is examined both in the linear and nonlinear regime. It is numerically shown that for rates of $10 \mathrm{~Gb} / \mathrm{s}$, the order of nanosecond delays can be achieved through the PCSW defect modes without excessive pulse broadening in the nonlinear regime. In the nonlinear case, it is shown that soliton pulses exhibit less broadening than pulses in the linear case. In comparing the linear and the non-linear case we consider launching pulses with the same initial full width at half maximum or the same RMS width. The influence of optical losses on the soliton pulse broadening factor is also incorporated and discussed providing a more practical perspective. The results demonstrate the potential of implementing a variety of linear and nonlinear signal processing applications in PCSWs, such as optical buffering.
\end{abstract}

Keywords: Photonic crystals, delay lines, optical waveguides, optical solitons

\section{INTRODUCTION}

All optical signal processing techniques may constitute a remedy for optoelectronic bottleneck encountered in modern optical networks [1]. However, several functionalities including optical buffering are very hard to implement in integrated form, necessitating the use of bulky components such us fiber delay lines [2]. Recent studies are supporting the increased attention to nanophotonic technologies, posing the idea of an all-optical buffering subsystem, using Photonic Crystals (PCs) [3]. PCs provide new means of manipulating light in a sub-wavelength scale opening up new paths in integrated optics design [4], [5]. PCs are formed by periodically modulating the refractive index of a material. Under certain conditions PC may exhibit photonic band gaps, i.e. frequency regions where no photons are allowed to propagate. By intentionally introducing a symmetry breaking, like a line defect, allows the formation of waveguides, which can guide light through sharp bends [6]. In these structures, propagation is supported by defect modes that appear inside the photonic band gap [3], [7] of the bulk crystal. This situation is unlike the index guiding mechanism which governs light propagation in conventional dielectric waveguides such as optical fibers [8]. PC slabs possess most of the optical properties of photonic crystals [6] and can be easily realized using standard lithographic techniques. PC slabs are periodic dielectric structures along two directions $(x$ and $y$ ) and use index guiding to confine light along the vertical direction $(z)$. Although there is a variety of line defects, that can be introduced inside the bulk PC, (Fig. 1a), most of the scientific attention is focusing on studying W1 photonic crystal slab waveguides (PCSW) (Fig. 1b), which are taken by simply removing a single row of holes in the bulk crystal. This is mostly due to the belief that these structures (Fig. 1a) sustain severe propagation losses. However some works seem to indicate that under certain design conditions such structures [9], [10] exhibit less propagation losses than the corresponding W1 waveguides. Propagation losses, under the light line, are primarily due to backscattering induced by fabrication imperfections. These losses can be combated by either improving the fabrication accuracy or applying some form of optical amplification.

Another fundamental limitation of PCSWs is the broadening of optical pulses due to the larger group velocity dispersion experienced with increasing group index, which is a result of the flattening of the guided mode's dispersion curve

Photonic Crystal Materials and Devices X, edited by Hernán Ruy Míguez, Sergei G. Romanov,

Lucio Claudio Andreani, Christian Seassal, Proc. of SPIE Vol. 8425, 842503 · C 2012 SPIE

CCC code: $0277-786 \mathrm{X} / 12 / \$ 18 \cdot$ doi: $10.1117 / 12.921468$ 
$\beta=\beta(\omega)$. Given a PCSW of length $L$, it is therefore not enough to simply calculate the achieved delay $T_{d}=L / v_{g}$ but one needs to consider both the level of pulse broadening and the amount of propagation loss. At higher power levels, nonlinear phenomena can also affect signal propagation which may turn out quite different than in the linear regime. For instance, when soliton pulses [11] propagate inside a Kerr nonlinear media, self phase modulation (SPM) can balance the GVD-induced broadening, in the absence of optical losses and higher order dispersion. The properties of both linear and nonlinear pulse propagation have been previously analyzed and compared in purely 2D PhC waveguides [12] and coupled resonator optical waveguides [13]. In this work, we compare the use of W1 PCSWs as delay lines in the linear and nonlinear regime, taking into account the full 3D nature of the structure. Propagation losses as well as pulse broadening are taken into account. We will show that under certain conditions nonlinear soliton propagation is better suited for slow light propagation in this type of structures.
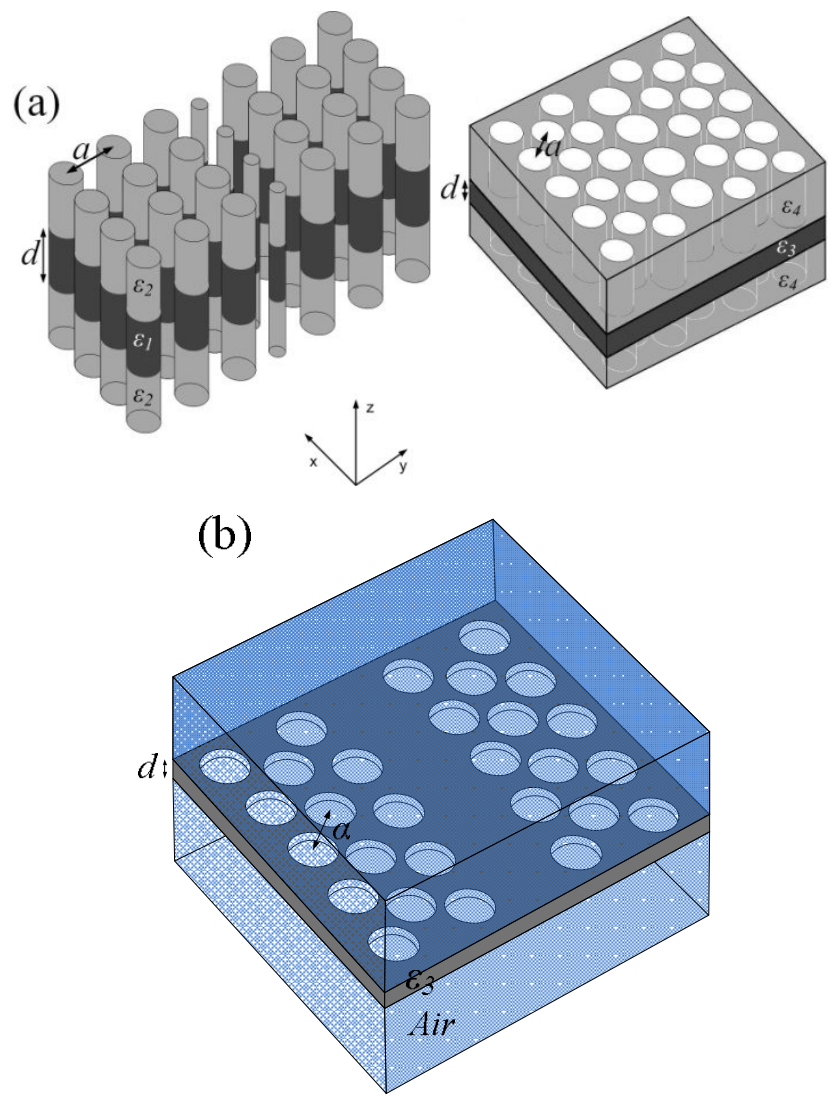

Figure 1. Different PCSW with introduced line defects a) a rectangular lattice of high index dielectric rods embedded in a low index material with a line defect of a single row with smaller radius $r_{d}<r_{a}$, and triangular lattice of low index dielectric rods embedded in a high index material with a line defect of a single row of holes with larger radius $r_{d}>r_{a}$ and b) a W1 PCSW membrane in air cladding.

\section{PROPAGATION MODEL}

\subsection{Electromagnetic Field Expansion and PCSW defect modes}

For narrowband signals, the total electromagnetic field inside the PCSW can be approximated by [14]:

$$
\mathbf{E}(\mathbf{r}, t) \cong A(x, t) \mathbf{e}\left(\mathbf{r}, \omega_{0}\right) e^{-j \omega_{0} t}+c . c .
$$




$$
\mathbf{H}(\mathbf{r}, t) \cong A(x, t) \mathbf{h}\left(\mathbf{r}, \omega_{0}\right) e^{-j \omega_{0} t}+c . c .
$$

where $A(x, t)$ is the slowly varying envelope function of the signal, $\mathbf{E}$ and $\mathbf{H}$ are the total electric and magnetic field inside the waveguide, $x$ is the propagation direction, $\omega_{0}=2 \pi c / \lambda_{0}$ is the central frequency of the signal, $\lambda_{0}$ the free space optical wavelength, $\mathbf{r}=(x, y, z)$ and c.c. stands for complex conjugate. The fields $\mathbf{e}(\mathbf{r}, \omega)$ and $\mathbf{h}(\mathbf{r}, \omega)$ are the electric and magnetic defect mode components. According to Bloch's theorem [15], these components can be expressed as $\mathbf{e}_{0}(\mathbf{r}, \omega) e^{j \beta(\omega) x}$ and $\mathbf{h}_{\mathbf{0}}(\mathbf{r}, \omega) e^{j \beta(\omega) x}$ where $\mathbf{e}_{\mathbf{0}}(\mathbf{r}, \omega)$ and $\mathbf{h}_{\mathbf{0}}(\mathbf{r}, \omega)$ are the periodic vector functions. The propagation constant $\beta(\omega)$ and modal fields $\mathbf{e}$ and $\mathbf{h}$ are determined by the following Hermitian eigenproblem for the magnetic field component [7]:

$$
\nabla \times\left(\frac{1}{\varepsilon_{L}(\mathbf{r})} \nabla \times \mathbf{h}(\mathbf{r}, \omega)\right)=\frac{\omega^{2}}{c^{2}} \mathbf{h}(\mathbf{r}, \omega)
$$

where $\varepsilon_{L}(\mathbf{r})$ is the linear part of the relative permittivity of the PCSW. By expanding $\mathbf{h}$ on a plane wave basis [15], eq. 3 is reduced into a matrix eigenvalue problem which can be solved using numerical techniques. We have implemented such a 3D plane wave expansion (PWE) mode solver based on the conjugate gradient minimization of the Rayleigh Ritz quotient [16]. For Kerr nonlinear media, the permittivity can be written as the sum of a linear and a nonlinear part:

$$
\varepsilon(\mathbf{r})=\varepsilon_{0}\left(\varepsilon_{L}(\mathbf{r})+\varepsilon_{N L}(\mathbf{r})|\mathbf{E}|^{2}\right)
$$

The nonlinear part of the permittivity can be expressed in terms of the nonlinear refractive index of the material $n_{2}(\mathbf{r})$ as [17]:

$$
\varepsilon_{N L}(\mathbf{r})=\frac{\varepsilon_{L}(\mathbf{r}) n_{2}(\mathbf{r})}{Z_{0}}
$$

where $Z_{0}$ is the free space impedance. Pulse broadening can be measured in terms of the root mean square (RMS) pulse width, [18] $\sigma(\mathrm{x})$, determined by:

$$
\sigma^{2}(x)=\frac{\int(T-\bar{T})^{2}|A(x, T)|^{2} d T}{\int|A(x, T)|^{2} d T}
$$

where $\bar{T}$ is the center of the pulse:

$$
\bar{T}=\frac{\int T|A(x, T)|^{2} d T}{\int|A(x, T)|^{2} d T}
$$

The broadening factor $\mathrm{BF}$ can be estimated through the following equation:

$$
B F=\frac{\sigma(L)}{\sigma(0)}
$$

where $L$ is the waveguide length and $\sigma(0)$ corresponds to the initial pulse width. Values of the broadening factor below 1.33 (implying a 33\% pulse broadening) are usually considered not to induce any serious ISI impairments [19]. 


\subsection{Estimation of BF in the Linear and Non-Linear Regime.}

In the linear regime $(\gamma=0)$, the pulse broadening is determined solely by the group velocity dispersion (GVD) and the third order dispersion (TOD) coefficients $\beta_{2}$ and $\beta_{3}$ respectively. Assuming a Gaussian pulse at the waveguide input, i.e. $A(0, T)=\exp \left(-T^{2} / 2 / T_{0}^{2}\right)$, then one can derive a closed form formula for the broadening factor [18] in eq. 8 :

$$
B F_{L}=\left(1+\left(z / L_{D 2}\right)^{2}+\frac{1}{4}\left(z / L_{D 3}\right)^{2}\right)^{1 / 2}
$$

where $L_{D 2}$ and $L_{D 3}$ are the dispersion lengths of the GVD and TOD respectively. In the nonlinear regime soliton pulses can be used to mitigate the effect of GVD induced broadening. In this case, broadening can still occur even in the lossless case because of the TOD. The soliton pulse shape depends on the sign of the GVD coefficient $\beta_{2}$ which as mentioned earlier can be either positive or negative, depending on the pulse launch frequency and waveguide parameters. If $\beta_{2}$ is positive (which is usually the case in the PCSWs considered here) then dark soliton should be launched in the structure, i.e. $A(0, T)=P_{0}{ }^{1 / 2} \tanh \left(T / T_{0}\right)$. At $T=0$ one obtains $A(0,0)=0$ while for $\left|T / T_{0}\right|>>1,|A(0, T)| \cong P_{0}{ }^{1 / 2}$ and hence this soliton pulse appears as a dip on a bright background. Pulse broadening in this case can be studied by numerically solving the propagation equation using the split step Fourier (SSF) method [18]:

$$
\frac{\partial A}{\partial x}+\frac{\Gamma}{2} A+\frac{j \beta_{2}}{2} \frac{\partial^{2} A}{\partial \mathrm{T}^{2}}-\frac{\beta_{3}}{6} \frac{\partial^{3} A}{\partial \mathrm{T}^{3}}=j \gamma|A|^{2} A
$$

where $\beta_{2}$ and $\beta_{3}$ are the coefficients of GVD and TOD respectively.

\subsection{Propagation Losses.}

PCSWs are intrinsically loss-less, however the low level of sophistication of integrated optical devices causes severe propagation losses [20], [21]. Several fabrication imperfections (sidewall roughness, dislocation, etc) can cause light scattering. Backscattering due to the coupling between the counter propagating mode and out-of-plane losses due to coupling with radiation modes are considered the main parameters of the propagation losses observed in experiments [22].. In Ref. [22] is shown that for standard W1 waveguides, propagation losses coefficient $a$ scale with respect to the group index $n_{g}$ according to the following equation:

$$
a=c_{1}^{\prime} n_{g}+c_{2}^{\prime} n_{g}^{2}
$$

where in $c_{1}^{\prime}$ and $c_{2}^{\prime}$ are including the out-of-plane and backscattering contribution respectively and $n_{g}=c / v_{g}$ is the group index. Backscattering is usually the dominant effect, indicating that in standard W1 waveguides, one can use a simpler $n_{g}{ }^{2}$ scaling for describing propagation losses, i.e.:

$$
a=a\left(n_{g 0}\right)\left(\frac{n_{g}}{n_{g 0}}\right)^{2}
$$

where $a\left(n_{g 0}\right)$ is the propagation losses for the fast light regime in $\mathrm{dB} / \mathrm{cm}$ and $n_{g 0}$ is the minimum group index achieved in the fast light regime.

\section{LINEAR OR NONLINEAR PROPAGATION IN PC-BASED DELAY LINES}

When designing a PC-based delay lime, one must take into account the amount of propagation losses and set a limit in the maximum allowable loss that can be experienced by the signal. This puts a limit in the maximum propagation distance $L_{w}$ and since the loss factor depends on the group index $n_{g}$, one also expects that $L_{w}$ will also depend on $n_{g}$. The maximum allowable loss $l_{\max }$ depends on the application at hand. If at some point, one wishes to use optical amplification to compensate for the optical losses, then $l_{\max }$ should not exceed $20 \mathrm{~dB}$, since this is a typical gain achieved 
by a semiconductor optical amplifier (SOA). If optical amplification is not an option, then $l_{\max }$ may have to be taken even smaller than that. In any case the propagation length is limited by

$$
L_{w} \leq L_{\mathrm{LOSS}}=\frac{l_{\max }}{a}
$$

where $a$ is the propagation losses described in $\mathrm{dBcm}^{-1}$. Fig. 2, illustrates the maximum propagation limit $L_{\mathrm{LOsS}}$ induced by the losses as a function of $n_{g}$ for various values of $a\left(n_{g 0}\right)$ in the case of a W1 PCSW for which $n_{g 0} \cong 5$. The values assumed for $a\left(n_{g 0}\right)$ are $2 \mathrm{dBcm}^{-1}, 5 \mathrm{dBcm}^{-1}$ and $10 \mathrm{dBcm}^{-1}$. It should be noted that certain loss levels allow for a propagation distance of the order of $1 \mathrm{dm}$. However, since the delay line should be realized in integrated form, such propagation lengths may be unrealistic. We therefore choose the propagation length $L_{w}$ to be fixed at $1 \mathrm{~cm}$ if $L_{\mathrm{LOSS}}$ exceeds $1 \mathrm{~cm}$. Otherwise we assume $L_{w}=L_{\mathrm{LOSs}}$.

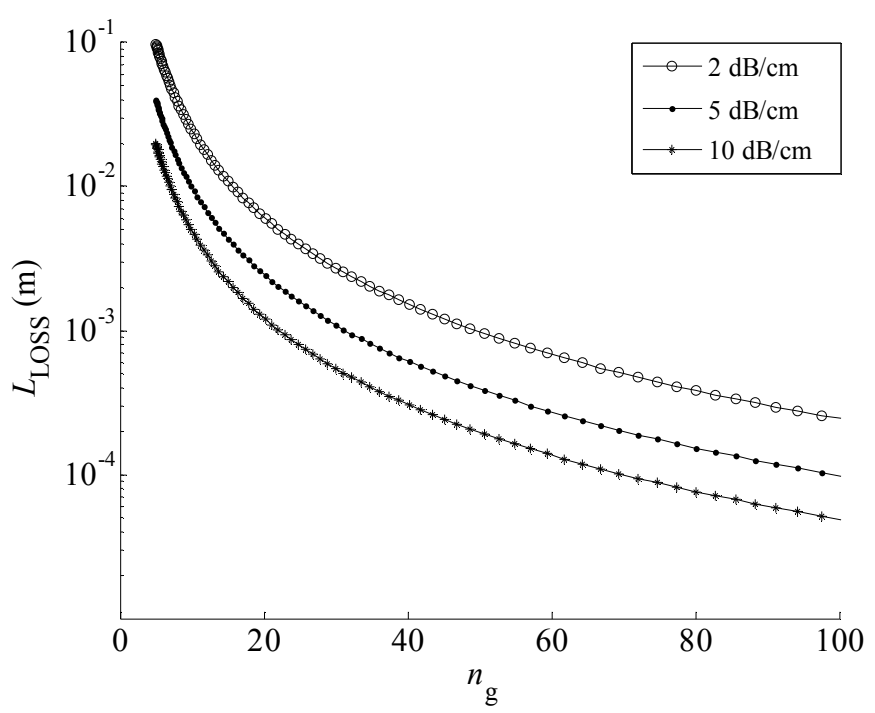

Figure 2. Propagation length limit as a factor of group index so that the output signal will be $20 \mathrm{~dB}$ debased.

Given the waveguide length $L_{w}$ we can calculate the broadening factor as a factor of the resulting delay time for several bit rates and various levels of fast light regime losses $a\left(n_{g 0}\right)$. In Fig. 3, we have calculated the broadening factor for a bit rate of $60 \mathrm{Gbps}$. We can see that the nonlinear soliton propagation by using the fundamental soliton power $\left(\mathrm{P}_{0}=\left|\beta_{2}\right| / \gamma \mathrm{T}_{0}{ }^{2}\right)$, sustain slightly less broadening than the linear Gaussian propagation. In this case we assume an even smaller optical loss margin of 10dB. In Fig. 4 the fundamental soliton power is shown for every $n_{g}$ assumed here. The results improves when solitons are launched with higher than the fundaental power $\left(P>P_{0}\right)$ as the soliton will experience an initial compression [24]. This improvement is less than $0.1 \mathrm{nsec}$ for a bit rate of $60 \mathrm{Gbps}$, translating in an additional 5 bits storage capacity from the linear case. 


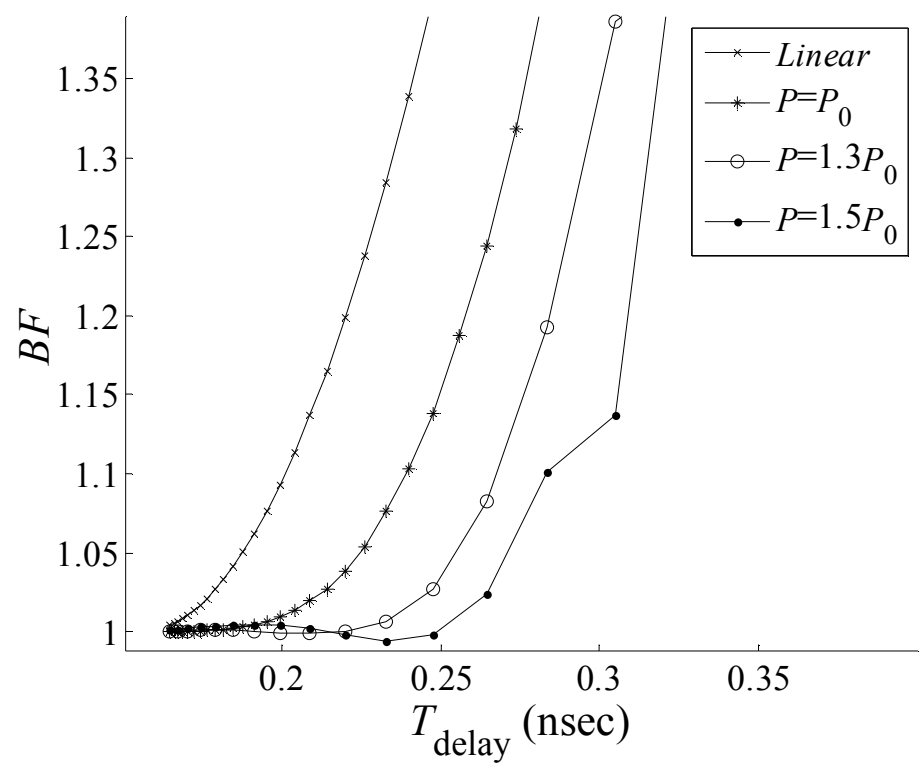

Figure 3. Broadening factor vs delay time in nsec at $60 \mathrm{Gbps}$. In this case we take into account fast regime losses $2 \mathrm{~dB} / \mathrm{cm}$ $\left(n_{g}=5\right)$ and waveguide length limited by $10 \mathrm{~dB}$ absorption.

In Fig. 5, the losses at the fast regime $\left(n_{g}=5\right)$ losses were set higher at $5 \mathrm{dBcm}^{-1}$. This figure shows that as we moving to more lossy cases the difference between the linear and nonlinear signal propagation is now smaller. As fabrication accuracy is increased in the future, nonlinear soliton signal propagation seems to be more suited for delay line applications.

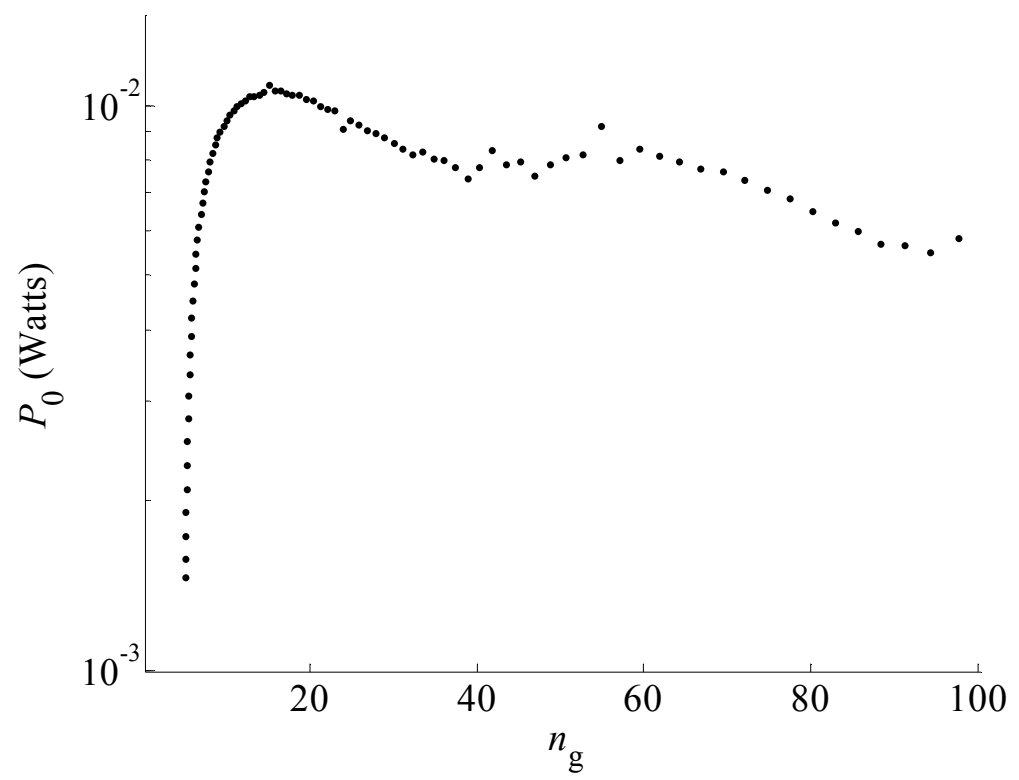

Figure 4. Fundamental soliton power $P_{0}$ vs $n_{g}$ for the bit rate of $60 \mathrm{Gbps}$. As seen here the calculated soliton power varies at a miliwatt power level for the majority of the group indices.

In Fig. 6 an intuitive comparison between the lossless linear and nonlinear case is shown for a lower bit rate of 10Gbps. Of course the lossless case cannot be achieved in any structure, however we can deduce that as the fabrication accuracy 
improves the closest to the lossless case we will be. As seen from Fig. 6 the delay limitations of such structures reaches an order of nanosecond delays for the bit rate of $10 \mathrm{Gbps}$.

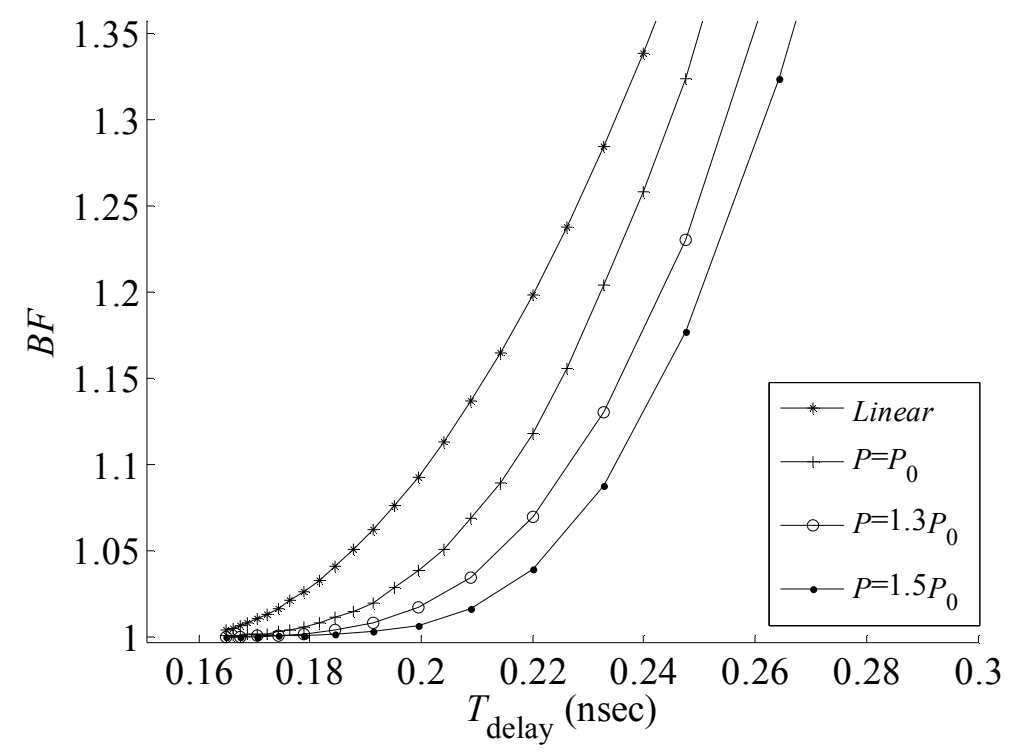

Figure 5. Broadening factor vs delay time in nsec at $60 \mathrm{Gbps}$. In this case we take into account fast regime losses $5 \mathrm{~dB} / \mathrm{cm}$ $\left(\mathrm{n}_{\mathrm{g}}=5\right)$ and waveguide length limited by $20 \mathrm{~dB}$ absorption.

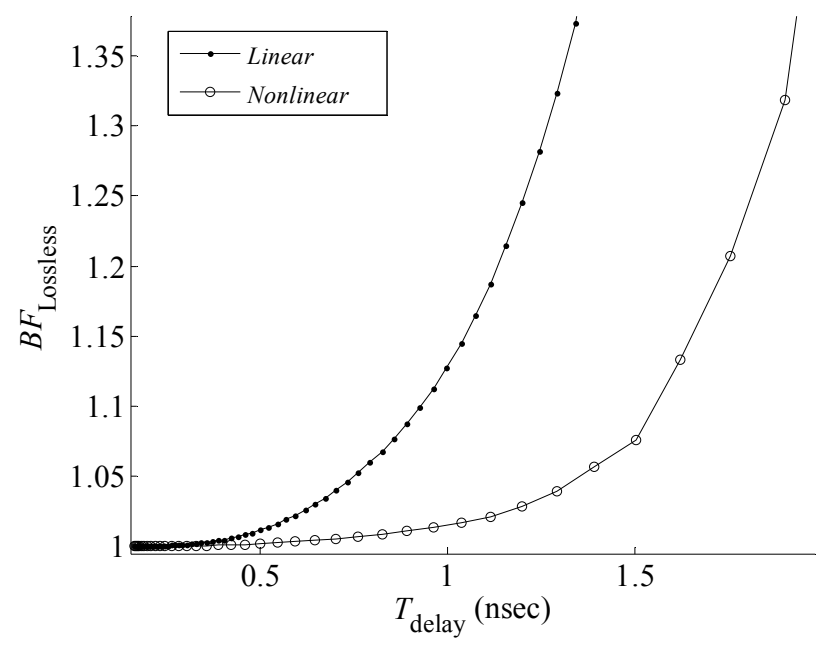

Figure 6. Broadening factor vs delay time in nsec at bit rate of $10 \mathrm{Gbps}$ assuming a lossless regime. It is expected that the nonlinear soliton propagation will sustain less broadening than the linear case.

\section{CONCLUSIONS}

In this paper, we studied and compared the linear and nonlinear propagation in PCSWs from a delay time point of view. The dispersion-induced broadening of an optical signal pulse as well as the propagation losses are taken into account. We have shown that the nonlinear case may provide important improvements to the delay time as long as the propagation losses are kept relatively low (corresponding to $2 \mathrm{dBcm}^{-1}$ in the fast light regime). As the fabrication 
accuracy improves over time and losses are reduced the nonlinear propagation may therefore find important applications in PCSW-based delay lines, improving the delay-bandwidth product achieved by such devices.

\section{REFERENCES}

[1] Dorren, H. J. S., Hill, M. T., Liu, Y., Calabretta, N., Srivatsa, A., Huijskens, F. M., H. de Waardt, and Khoe, G. D., "Optical packet switching and buffering by using all-optical signal processing methods," J. Lightw. Technol., vol. 21, no.1, pp. 2-12, Jan. 2003.

[2] Zhong, W. D. and Tucker, R. S., "A new wavelength routed photonic packet buffer combining traveling delay lines with delay-line loops,” J. Lightw. Technol., vol.19, pp. 1085-1092, Aug. 2001.

[3] Kitayama, K., Kubo, T., Takahashi, R., Matsuo, S., Arakawa, S., Murata, M., Notomi, M., Nozaki, K., and Kato, K., "All-optical RAM Buffer Subsystem Demonstrator," in Optical Fiber Communication Conference, OSA Technical Digest (CD) (Optical Society of America, 2011), paper OMK

[4] Fidaner, O., Demir, H. V., Sabnis, V. A., Zheng, J. F., Harris, J. S., Miller, Jr., and Miller, D. B., "Integrated photonic switches for nanosecond packet-switched optical wavelength conversion," Opt. Exp., vol. 14, pp. 361368, 2006

[5] Yanik, M. F., and Fan, S., "Stopping light all optically," Phys. Rev. Lett., vol. 92, no. 8, Feb. 2004, 083901.

[6] Johnson, S. G., Fan, S., Villeneuve, P. R., and Joannopoulos, J. D., "Guided modes in photonic crystal slabs", Phys. Rev. B, vol. 60, pp. 5751-5758, 1999.

[7] Johnson, S. G., Villeneuve, P. R., Fan, S., and Joannopoulos, J. D., "Linear waveguides in photonic-crystal slabs," Phys. Rev. B, vol. 62, no. 12, pp. 8212-8222, 1999.

[8] Collin, R. E., Field Theory of Guided Waves, $2^{\text {nd }}$ ed. Piscataway, NJ: IEEE Press, 1990.

[9] Gerace, D. and Andreani, L.C., "Effects of disorder on propagation losses and cavity Q-factors in photonic crystal slabs," Photonics and Nanostructures Fundam. Appl. 3, 120-128, 2005.

[10] Kok, A. A. M., van der Tol, J. J. G. M., Baets, R., and Smit, M. K., "Reduction of Propagation Loss in PillarBased Photonic Crystal Waveguides," J. Lightwave Technol. 27, 3904-3911 (2009).

[11] Combrie, S., De Rossi, A., Tran, Q. V., Benisty, H., "GaAs photonic crystal cavity with ultrahigh Q: microwatt nonlinearity at $1.55 \mu \mathrm{m}$," Opt. Lett., vol. 33, pp. 1908-1910, 2008.

[12] Theocharidis, A., Kamalakis, T., Chipouras, A., and Sphicopoulos, T., "Linear and nonlinear optical pulse propagation in photonic crystal waveguides near the band edge," IEEE J. Quantum Electron., vol. 44, no. 11, pp. 1020-1027, Nov 2008.

[13] Neokosmidis, I., Kamalakis, T., and Sphicopoulos, T., "Optical delay lines based on soliton propagation in photonic crystal coupled resonator optical waveguides," IEEE J. Quantum Electron., vol. 43, no. 7, pp. 560-567, Jul. 2007.

[14]Kamalakis, T. and Sphicopoulos, T., "A new formulation of coupled propagation equations in periodic nanophotonic waveguides for the treatment of Kerr-induced nonlinearities," IEEE J.Quantum Electron., vol. 43, no. 10, pp. 923-933, Oct. 2007.

[15] Sakoda, K., Optical Properties of Photonic Crystals. Berlin, Germany: Springer-Varlag, 2001.

[16] Johnson, S. G. and Joannopoulos, J. D., "Block-iterative frequency domain methods for Maxwell's equations in a planewave basis," Opt. Exp., 8, 173, 2001.

[17]Fujisawa, T. and Koshiba, M., "Finite-element mode solver for nonlinear periodic optical waveguides and its application to photonic crystal circuits," J. Lightw. Technol., vol. 23, no. 1, pp. 382-387, Jan. 2005.

[18] Agrawal, G. P., Non-Linear Fiber optics. New York: Academic, 2001.

[19] Ramaswami, R. and Sivarajan, K. N., Optical Networks: A Practical Prespective. San Mateo, CA: Morgan Kaufman, 1998.

[20] Andreani, L. C., and Gerace, D., "Light-matter interaction in photonic crystal slabs," Phys. Status Solidi B 244, 3528-3539 (2007).

[21] White, T. P., O'Faolain, L., Li, J., Andreani, L. C., and Krauss, T. F., "Silica-embedded silicon photonic crystal waveguides," Opt. Express 16, 17076-17081 (2008)

[22] O'Faolain, L., Schulz, S. A., Beggs, D. M., White, T. P., Spasenovic, M., Kuipers, L., Morichetti, F., Melloni, A., Mazoyer, S., Hugonin, J. P., Lalanne, P., and Krauss, T. F., "Loss engineered slow light waveguides," Opt. Express 18, 27627-27638 (2010). 
[23] Li, J., White, T. P., O’Faolain, L., Gomez-Iglesias, A., and Krauss, T. F., "Systematic design of flat band slow light in photonic crystal waveguides," Opt. Express 16(9), 6227-6232 (2008).

[24] Colman, P., Husko, C., Combrié, S., Sagnes, I., Wong, C. W. and De Rossi, A., "Temporal solitons and pulse compression in photonic crystal waveguides," Nature Photonics 4, 862 (2010).

[25] Notomi, M., Tanabe, T., Shinya, A., Kuramochi, E., Taniyama, H., Mitsugi, S., and Morita, M., "Nonlinear and adiabatic control of high-Q photonic crystal nanocavities," Opt. Express 15(26), 17458 (2007).

\section{Acknowledgments}

This research has been co-financed by the European Union (European Social Fund - ESF) and Greek national funds through the Operational Program "Education and Lifelong Learning" of the National Strategic Reference Framework (NSRF) - Research Funding Program: Heracleitus II. Investing in knowledge society through the European Social Fund. 